in position, and unaltered in shape. A thoracoscope was inserted in the fourth 'space, in the mid-axillary line, and a bluish-purple tumour could be seen, with fine veins on its surface arising in the posterior mediastinum from behind the great vessels and extending almost up to the arch of the subclavian artery and completely independent of the lung. A painting was made by Mr. Shiells (Plate, Fig. 2).

Four days later the patient was operated on by $\mathrm{Mr}$. Holmes Sellors, and so far as was possible the tumour was removed. It was filled with a chocolate-coloured thick creamy substance. This was sucked out and finally mopped dry with gauze. The portion of the wall of the tumour under the great vessels had to be left behind: it was wiped clean and swabbed with formalin. The patient made an uninterrupted recovery.

The report on the tumour was as follows: "The cyst wall consists in great part of vascular fibrous tissue with a striking amount of lymphoid tissue disposed mainly around the vessels. In some parts large thin-walled blood spaces are seen and haemorrhage has occurred. Other components of the cyst wall are fat, bundles of muscle fibres, ciliated columnar epithelium, and acinous glands. No ectodermal elements have been recognized, and no cartilage or bone." The blow from the tennis ball had possibly caused a haemorrhage into the teratoma.

\section{Case IV}

A man aged 33 complained of pain in the right side of the chest. Sixteen months previously he had had pain in the region of the dorsal spine radiating to the right costal margin anteriorly. For this condition he was admitted into a hospital in the North, where, after complete investigation, his appendix was removed; the pain, however, still persisted. Because of this he was again examined, and a skiagram of his chest revealed a mass in the right lung. He was admitted into the London Chest Hospital.

Physical examination was negative, except for some change in the percussion note at the right base posteriorly. The Wassermann reaction and the Casoni test were negative. The skiagram revealed a single spherical mass with smooth, sharply defined borders the size of an orange, situated in the right lower lung field (Plate, Fig. 3). A true lateral skiagram showed the mass to be lying close to the vertebral column (Plate, Fig. 4). Bronchoscopic examination was negative. An artificial pneumothorax was induced; the lung appeared to collapse away from the tumour, suggesting an innocent condition. A thoracoscope was introduced into the fifth space in the mid-axillary line. The mass was seen to be reddish yellow in colour, arising from the chest wall close to the vertebral column. It appeared to have a well-defined capsule. The lung was adherent to the growth at one point only, but it was infiltrated with multiple yellow sago-like nodules. Adjacent to the tumour, on the chest wall, were three secondary masses the size of a pea.

A section of the tumour was taken by means of forceps introduced through a second cannula; no undue bleeding was caused by this procedure. The section was reported by Drs. Gloyne and Page as a mixed-cell sarcoma, ? malignant change in a neurofibroma. In view of these findings a radical operation was regarded as impossible, and the patient was referred for deep $x$-ray therapy.

A recent necropsy provides another illustration of the potentialities of the method.

A woman was admitted to another hospital with a massive pleural effusion. The spleen was enlarged and a mass could be felt in the epigastrium. All the usual tests were made, but without result, and the differential diagnosis was malignant growth or lymphadenoma. An exploratory laparotomy had been advocated by two or three physicians as a diagnostic measure. Before this was done, however, the patient died. The post-mortem examination revealed a huge malignant mass behind the stomach, large secondary growths in the spleen, and metastases in many other parts: but, what is of interest in connexion with this communication, there were a number of raised white nodules the size of half a pea-nut on the parietal pleura. It would have been a simple matter to replace the fluid by air through the thoracoscopic cannula, to insert the telescope, see the nodules, and remove a portion for section, whereas the proposed alternative would have been highly dangerous.

As regards technique, one of us (F. G. C.) uses a singlecannula method with a direct-vision telescope. Through this single cannula all instruments can be inserted, including the biting forceps and a diathermy knob for the arrest of haemorrhage, if any. The other employs the two-cannula technique when removing a piece of the growth for section.

\section{Conclusion}

The cynics have suggested, occasionally with some truth, that modern methods of investigation are more distressing and dangerous to the patient than the disease itself; but this criticism cannot seriously be advanced in the case of an exploratory thoracoscopy; for in skilled hands the patient should feel nothing more than the first hypodermic injection of the local anaesthetic, and it is clear that this investigation, as illustrated by Cases II and IV, may save the patient an unnecessary thoracotomy. If a pleural effusion is present, then the thoracoscopy can be carried out at the same time as the aspiration provided the paracentesis is accomplished through a thoracoscopic cannula, a procedure which gives more efficient evacuation of the fluid than an ordinary needle, with no more discomfort.

With ordinary aseptic precautions an exploratory thoracoscopy should be without risk to the patient. This has been our experience with a large number of cases.

\section{CONGENITAL ATRESIA OF THE OESOPHAGUS}

\author{
BY
}

\author{
J. W. D. BULL, M.B., M.R.C.P. \\ Medical Registrar, St. George's Hospital
}

(With Special Plate)

The following case is recorded not on account of its rarity but because the condition has been so seldom mentioned in the English literature.

\section{Case History}

The patient was a female child born on August 23, 1937, of healthy parents. The mother had had three pregnancies: boy, who died at the age of 3 of pneumonia; a miscarriage ; and the patient. Delivery in the present case, in the maternity ward of St. George's Hospital, was spontaneous and normal. Excessive liquor amnii was noted. There was some difficulty in reviving the baby, as she seemed choked with mucus. The excess of mucus continued, but nothing alarming was observed until the second day, when the mother's milk had come in. On being put to the breast the baby sucked easily, but in a few minutes choked, and turned blue in the face. The nurse rescued it, and sucked out the milk and mucus from its mouth. The same result was obtained at the next feed. Mucus in the mouth increased in quantity, and, apart from the attempted feeding, the child had attacks of cyanosis, which were relieved by clearing the throat.

On the second evening Dr. T. F. McNair Scott examined the child. It was lying in the cot, breathing with some difficulty and through a great deal of moist mucus in the mouth and nose. It was normally developed and nourished, but moderately dehydrated. The skin was clear. No glands were palpable. Examination of the head revealed no craniotabes. The anterior fontanelle measured about $1 \frac{1}{2}$ by $1 \frac{1}{2}$ inches, being depressed, and the posterior fontanelle one finger-tip. 
The eves, mouth, nose, ears, neck, and heart were normal. There was no impairment of percussion note anywhere in the lungs. Breath sounds were transmitted mainly to the interscapular region. Nothing abnormal was found in the abdomen. Respiratory sounds were clearly transmitted to the stomach region. The genitalia were those of a normal immature female and the limbs were normal. Meconium was being passed.

From the history of excessive mucus and inability to take food without choking, a diagnosis of congenital absence of the oesophagus was made.

\section{INVESTIGATIONS}

1. Radiological.- On examining the baby under the screen it was immediately noticeable that the stomach contained no air. After the child had sucked the bottle containing milk and a barium salt, some air did appear in the stomach. The opaque meal was seen to enter, and to distend a blind point of the oesophagus which ended at the level of the second thoracic vertebra. The baby vomited soon after, and became very choked up. A radiograph confirmed the condition (Plate, Fig. 1). In addition a hemivertebra was present between the fifth and seventh thoracic, and the sixth right rib was absent.

2. Examination of meconium by staining a smear of it with gentian-violet and decolorizing with acid alcohol-Farber's test (1933)-revealed the presence of squamous epithelial (amniotic) cells as in normal meconium. This indicated that there must be a communication between the mouth and the intestine. Since the proximal part of the oesophagus ended in a blind pouch there must have been a communication hetween the trachea and the stomach.

\section{COURSE}

In view of the above findings it was decided that no operative intervention was indicated. All feeding by mouth was stopped, and the baby was kept hydrated by the introduction of from 60 to $120 \mathrm{c} . \mathrm{cm}$. of 5 per cent. glucose and saline intraperitoneally twice daily. The cot was kept at an angle of about 30 degrees, the head being down. Mucus continued to be quite profuse, and the baby had several attacks of cyanosis. With the aid of suction, and 1/200 grain of atropine when considered necessary, this was overcome. The baby remained comfortable, and slept most of the time.

The physical signs did not change much from those originally described until the day before death. The lungs remained resonant to percussion, but adventitious sounds developed, mainly rhonchi and coarse rales. On that day, following the intraperitoneal injection, quite profuse bleeding occurred from the puncture wound. Local pressure and thromboplastin were used, but the ooze continued. About thirty-six hours afterwards, with cessation of bleeding, the baby died. The blood taken after death was sterile. It was noted, however, that clotting did not occur.

Haemorrhage occurring in a newborn infant associated with delayed or absent clotting suggested a diagnosis of haemorrhagic disease of the newborn. The unusual feature here was that the onset of the bleeding was delayed to the eighth day in the absence of a septicaemia.

Before performing the necropsy it was decided to inject lipiodol into the trachea to see if the suspected tracheooesophageal fistula could be demonstrated. This was seen on the screen, and the lower portion of the oesophagus was shown to be leaving the trachea about five millimetres above the bifurcation. The lipiodol filled the bronchial tree and passed to the lower end of the oesophagus (Plate, Fig. 2). It was prevented from entering the stomach by an air-lock, as was afterwards discovered at necropsy.

\section{Necropsy}

The body was that of a somewhat wasted child. There were numerous puncture marks on the abdominal wall. The congenital atresia of the oesophagus was confirmed, as was also the tracheo-oesophageal fistula (Plate, Fig. 3-St. George's Hospital Pathological Museum). A window has been cut in the anterior border of the trachea to show the fistula. There was no fibrous communication between the two portions of the oesophagus. The stomach contained no food, only meconium. The rest of the gut was normal, as were the biliary system, liver, and spleen. The heart and great vessels were also normal, but the ductus arteriosus was still patent. The suprarenals were normal in size, shape, and position. The right kidney and ureter were normal. The left kidney was extremely atrophic, and the pelvis was very dilated and was as large as the kidney itself. The ureter, which entered the bladder, was very short, and as a result the left kidney was within the pelvis minor. The bladder and urethra were normal. The genital system was also abnormal on the left side. The left cornu of the uterus had not developed at all, and the left round ligament joined the right cornu at its cervical end. The medial end of the left Fallopian tube terminated blindly at some distance from the uterus. The left ovary appeared normal. The drawing here reproduced shows the condition

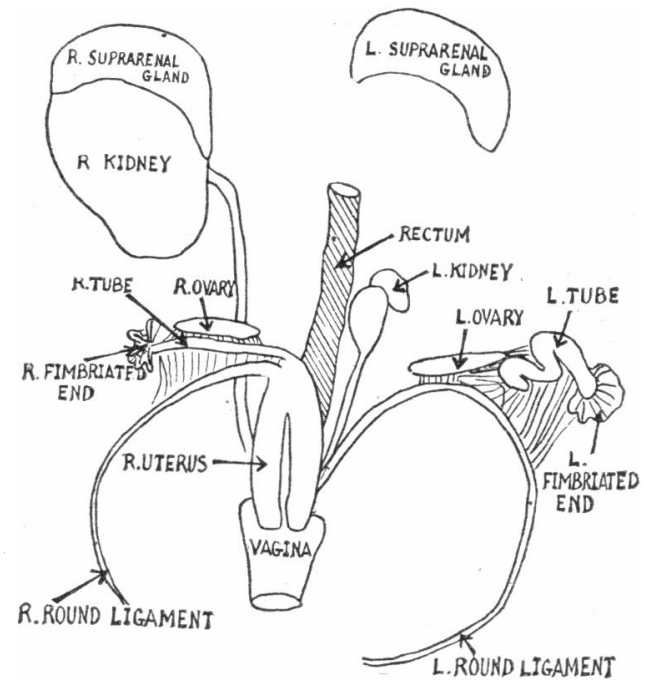

diagrammatically, the bladder being omitted for the sake of simplicity. No other abnormalities were found.

\section{Cases in the Literature}

The first published case of this condition was observed by Thomas Gibson (1703), a Fellow of the College of Physicians, in 1679 , and it is noteworthy that his clinical description was faultless. He afterwards performed a necropsy, on which the diagnosis was made. Since then, however, the condition has received scant attention in the English literature, and it has been left to the American schools particularly, and to some extent the European schools, to draw attention to it.

Plass (1919) collected 136 cases. He showed that about 30 per cent. had other congenital abnormalities. Rosenthal and Himmelstein (1932) noted 255 cases in the literature, and 204 of these had tracheo-oesophageal fistulae. Recently at least ten cases have been reported annually. The various types of abnormality have been classified by McClellan and Elterich (1934). They describe twelve different types, which are clearly understood when one considers the development of the oesophagus and trachea. Keith and Spicer (1907) described the embryology, and Keith (1910) illustrated it by cases in a later publication. They state that in the $3.2 \mathrm{~mm}$. embryo (third week) the floor of the anterior part of the primitive oesophagus becomes evaginated and forms the trachea. In the $12.5 \mathrm{~mm}$. embryo (fifth week) the tracheo-oesophageal septum has completely formed.

The symptomatology as described in the case above is quite typical. Few live more than a week, and none has lived more than a month. Death is nearly always due to bronchopneumonia. 


\section{Treatment}

Gastrostomies have been performed repeatedly; but, unless the cardiac end of the stomach is tied, the food flows backwards up the oesophagus into the trachea, except in the very few cases where there is no tracheo-oesophageal fistula. It has been the hope of some surgeons to keep the patients alive long enough to perform an intrathoracic plastic operation at a later date. This seems a forlorn hope, and it is questionable whether it would be justified even if successful, for so many cases are associated with multiple congenital lesions.

\section{Summary}

1. A report of a case of congenital atresia of the oesophagus with tracheo-oescphageal fistula is given.

2. The indications are that the anomaly occurs much more often than is generally believed.

3. The anomaly is nearly always associated with other congenital lesions.

4. It produces a quite characteristic syndrome, which if kept in mind makes a clinical diagnosis relatively simple.

5. Auxiliary methods of diagnosis are mentioned.

6. Death has occurred within four weeks in every recorded case.

I should like to express my thanks to Dr. T. F. McNair Scott, recently paediatrician to St. George's Hospital, for allowing me to record this case.

\section{REFERENCES}

Farber, S. (1933). J. Amer. med. Ass., 100, 1753.

Gibson, T. (1703). Anat. of Human Bodies Epitomized, 6th ed., p. 240, London,

Keith, A. (1910). British Medical Journal, 1, 301.

- and Spicer, J. E. (1907), J. Anat Physiol 41, 52.

McClellan, R. H., and Elterich, T. O. (1934). Arch. Pediat., 51, 171.

Plass, E. D. (1919). Johns Hopk. Hosp. Bull., 18, 259.

Rosenthal, A. H., and Himmelstein, U. (1932).' Arch. Pediat., 49, 444.

\section{THE BACTERIOSTATIC EFFECTS OF SULPHONAMIDE-P, SOLUSEPTASINE, AND M \& B 693 \\ BY}

\section{B. G. MAEGRAITH, M.B., B.Sc., D.Phil. \\ AND}

R. L. VOLLUM, D.Phil.

(From the Sir William Dunn School of Pathology, Oxford)

The bacteriostatic properties of these three drugs on Streptococcus viridans and Staphylococcus aureus were examined by the slide method of Wright, modified by Fleming $(1924,1938)$. A different technique, described below, was necessary in the cases of Neisseria gonorrhoeae and $N$. meningitidis, as these organisms did not show sufficiently differentiated colonies for counting. The drugs were dissolved in saline and kept made up in dilutions of 1 in $1, \theta 00$, sterilized by heating to $60^{\circ} \mathrm{C}$. for one-half to one hour.

\section{Streptococcus viridans and Staphylococcus aureus}

\section{TECHNIQUE}

The tests were set up in the following manner. First a suspension of a fresh culture of the organism in sterile saline was prepared and matched to correspond in density with Brown's No. 1 tube. Human blood was then withdrawn and defibrinated by shaking with glass beads. Half of the blood was separated and forced five to seven times through cotton-wool to reduce the number of leucocytes present. In this way the white cell count was lowered to 500 to 1,000 per c.mm. and most of the polymorphs were removed. Blood so treated is referred to below as "deleucocyted." Defibrinated blood was found to be slightly deleucocyted, the white cell count after defibrination falling from about 7,000 to 5,000 per c.mm., but many polymorphonuclear cells were still present.

Mixtures were then made of one volume of suspension of the organisms to three volumes of defibrinated and of deleucocyted blood. Equal volumes of the blood-organism mixtures and the dilution of the antiseptic were then mixed and slides set up as described by Fleming. Three dilutions of each chemical substance were employed, and the final dilutions in the compartments of the slides were 1 in 2,000, 1 in 20,000, and 1 in 60,000. Prepared slides were incubated at $37^{\circ} \mathrm{C}$. in moist conditions for twentyfour hours, and after incubation colonies were counted under the low power of a microscope.

\section{RESULTS}

Streptococcus viridans.-Three strains isolated from patients' throats were examined, and the results in all three examinations showed that the drugs affected the growth of the organisms, but only in the presence of white blood corpuscles, including polymorphs. A typical result of a count of Str. viridans is shown in Table I.

TABLE I.-Effect of Drugs on Str. viridans

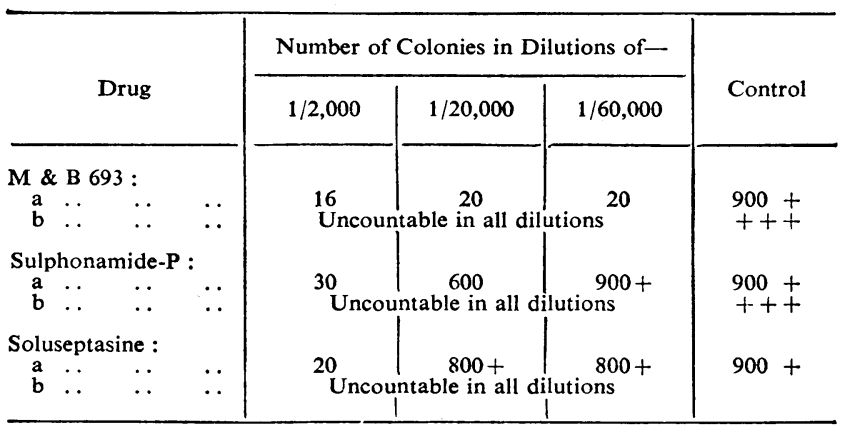

$a=$ mixed with defibrinated blood $; b=$ mixed with deleucocyted blood + sign after a number indicates the presence of very small colonies not counted in the figure quoted.

Similar results were obtained for the other strains of Str. viridans. In the presence of defibrinated blood containing normal numbers of white cells it will be seen that M \& B 693 is a very efficient bacteriostatic agent, even at a dilution of 1 in 60,000 , whereas the other two drugs are efficient at a dilution not greater than 1 in 2,000. In deleucocyted blood none of the drugs has much bacteriostatic effect, although the colonies appear much smaller in the high concentrations than in the controls, which contained no drugs.

Staphylococcus aureus.-None of the drugs appeared to have the slightest effect on the growth of this organism. Two strains of staphylococcus, one freshly isolated from a boil, were examined, and in both cases the growth after twenty-four hours was confluent and general in all slides.

\section{Neisseria gonorrhoeae and N. meningitidis} TECHNIQUE

These organisms were examined by the slide method, but this was abandoned after a few experiments, as colony formation was poor, making counting very difficult. The following method was then devised. Mixtures of one volume of suspensions of fresh cultures of the organisms and three volumes of defibrinated and deleucocyted blood 
J. W. D. BULL : CONGENITAL ATRESIA OF THE OESOPHAGUS

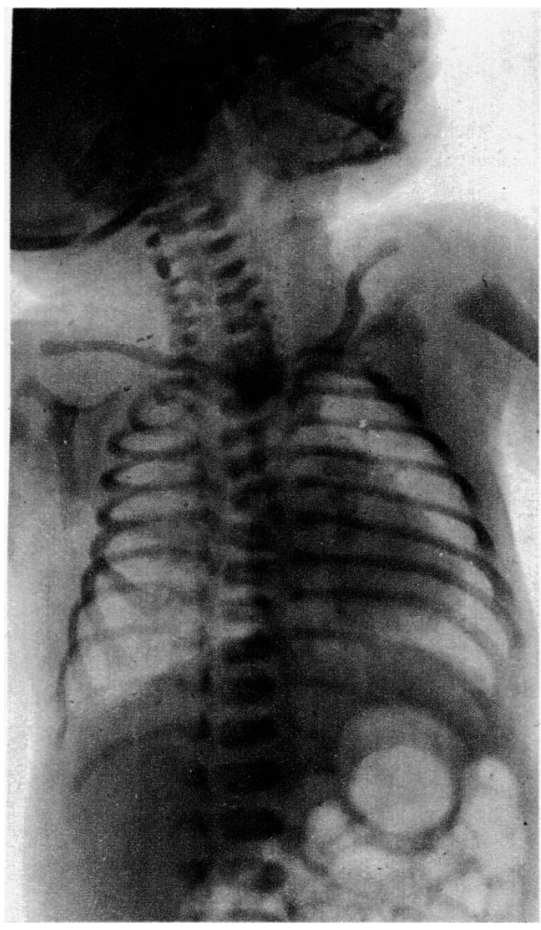

Fio. 1.-Radiograph taken in life.

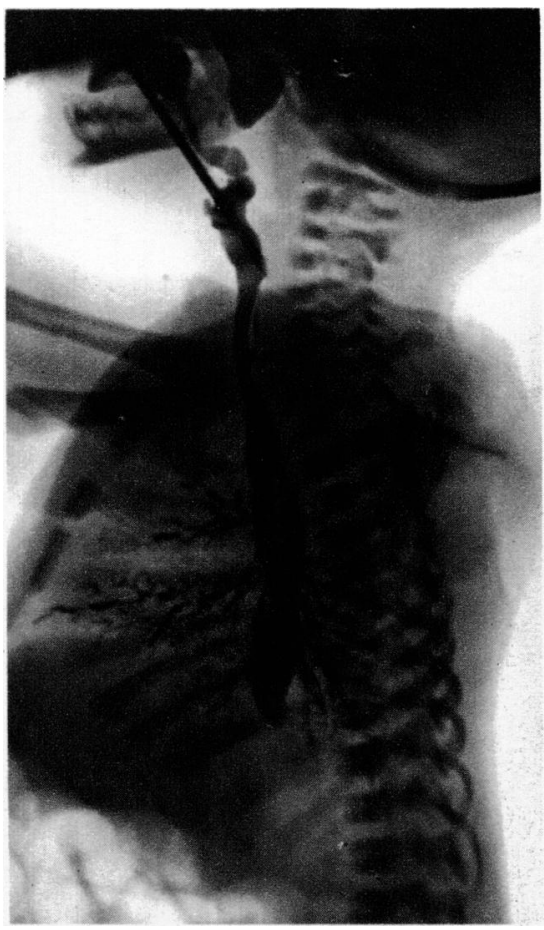

Fia. 2.-Radiograph taken after death, showing lipiodol injected into the trachea filling the oesophagus and outlining the bronchial tree.

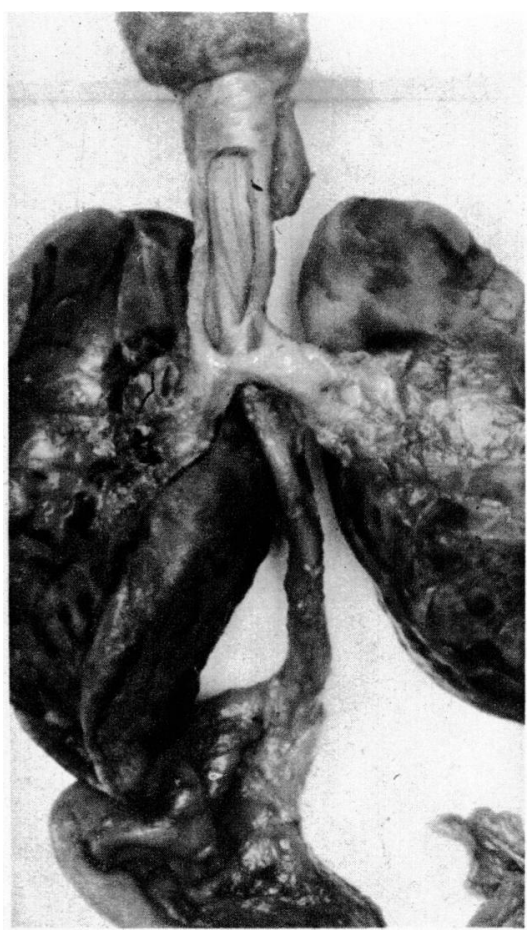

Fic. 3.-Specimen showing the congenital atresia of the oesophagus and the tracheooesophageal flstula. OVARIAN CYSTS

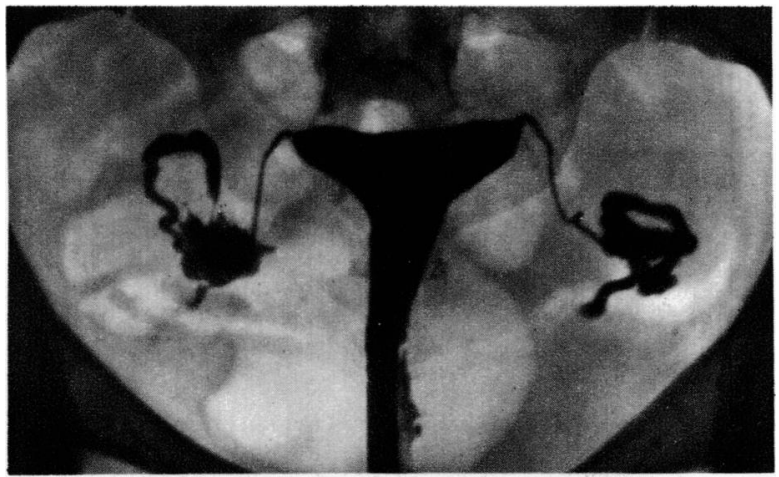

Fio. 1.-Uterus and tubes normal.

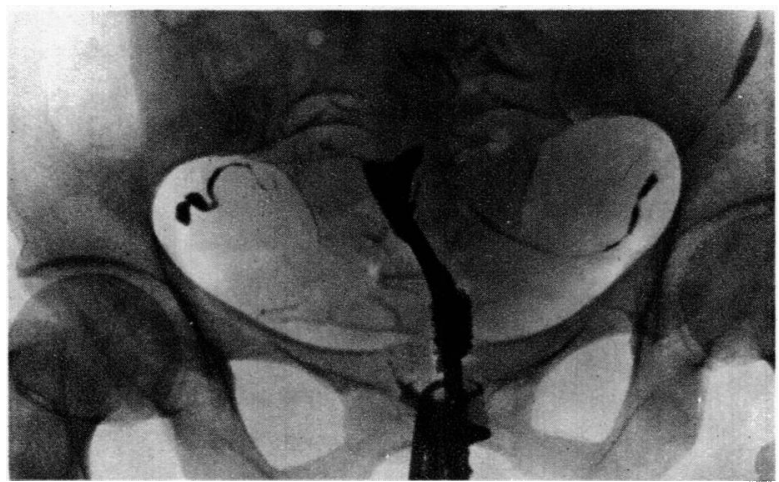

FiG. 2.-One tube considerably elongated compared with the other.
HENRY POSTON : BILATERAL DIGITAL EPIPHYSEAL DEFORMITY

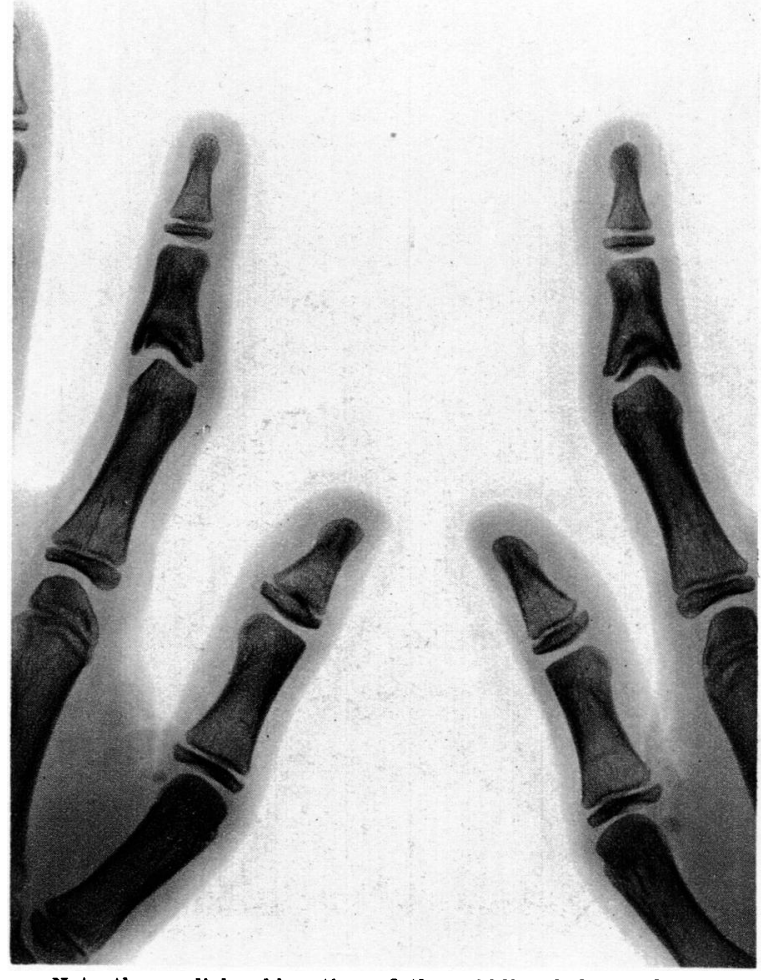

Note the medial subluxation of the middle phalanx of each index finger and the peculiar angulation of the epiphyses. 